\title{
Determinants of smoking behaviour among adolescents in Semarang, Indonesia
}

\author{
Bart Smet, Lea Maes, Linda De Clercq, Kristiana Haryanti, Rachmad Djati Winarno
}

Department of Behavioural Therapy and Psychological Consultation, University of Ghent, Ghent, Belgium B Smet

L De Clercq

Department of Public Health L Maes

Faculty of Psychology, Catholic University Soegijapranata, Semarang, Indonesia K Haryanti

R Djati Winarno

Correspondence to: L Maes, Department of Public Health, University Hospital, Block A, De Pintelaan 185, B-9000 Ghent, Belgium

lea.maes@rug.ac.be

Received 23 April 1998 and in revised form 2 February 1999. Accepted 17 February 1999

\begin{abstract}
Objectives-To determine the smoking prevalence and to examine the determinants of smoking behaviour among male adolescents in Semarang, Indonesia. Design-A random sample of schools in Semarang (population 1.5 million) was obtained using a stratified sampling procedure (strata based on type of school and district). A total of 149 schools were selected (response rate $72 \%$ ). Within the schools 186 classes were selected, targeting the 11, 13, 15, and 17 year olds. An anonymous, self administered questionnaire was filled in by all students present at the day of the survey (total sample size 6276).

Outcome measures-Smoking prevalence, exposure to family and peer smoking, and other variables that may be related to smoking. Logistic regression was used to examine the determinants of smoking behaviour. Only male students were included in the analysis.

Results-Smoking increased dramatically between the ages of 11 and 17, from $8.2 \%$ to $38.7 \%$. The variance explained by the regression model increased from $19.8 \%$ for 11 year olds to $53 \%$ for 17 year olds. The smoking behaviour of best friends was the most powerful determinant of smoking, and this was consistent across the age groups. Best friends' attitudes towards smoking and older brothers' smoking behaviour were also important determinants of smoking.

Conclusions-Smoking prevalence among male adolescents in Semarang, Indonesia is high. Effective smoking prevention programmes should take into account the dominant influence of peers in the onset and maintenance of smoking behaviour. In general, school related items had a less important role in predicting smoking behaviour than expected.

(Tobacco Control 1999;8:186-191)
\end{abstract}

adolescents; smoking prevalence; smoking initiation predictors; Indonesia

\section{Introduction}

The initiation and predictors of smoking by adolescents, although well documented in the West, have been less well studied in the Asia Pacific region. ${ }^{1}$ Nevertheless, smoking is still on the increase, especially in developing countries. The World Health Organisation estimates that about $50 \%$ of men and $8 \%$ of women in developing countries are smokers. ${ }^{2}$ A study on the prevalence of cigarette smoking in a rural area of west Java showed that more than $80 \%$ of men were cigarette smokers. Cigarette smoking is a practice that is widely accepted among men without limitation. By contrast, under $5 \%$ of adult females are current smokers. As is found in many other developing countries, cigarette smoking is not well established among women. ${ }^{3}$

Although many surveys in Indonesia confirm that smoking among children of school age is relative high, ${ }^{4}$ results vary, probably because of different research design, methods, and age groups, rather than because of different smoking behaviour.

Because of the practical and theoretical importance of the problem, we need information about the processes that affect the probability of early tobacco use. ${ }^{5}$ The ability to identify groups of children at risk of initiating smoking may prove useful in developing effective smoking prevention programmes. ${ }^{6}$ Many studies have examined predictors of smoking among adolescents. Environmental variables are often found to be the strongest predictors. In an overview by Reid et $a l^{7}$ on smoking in young people in western countries, it was concluded that such factors as availability of cigarettes, the perception that tobacco use is the norm, peer and sibling attitudes, and lack of parental support were associated with adolescent smoking. The influence of parental smoking seemed less clear. The same overview stated that behavioural predictors of smoking include low academic achievement, rebelliousness, alienation from school, and lack of skills to resist offers of cigarettes. Personal risk factors include low self esteem and the belief that smoking confers future advantages in social life. Cross-national data from the WHO survey on health behaviour in school-aged children (HBSC) - the study that was also used for this paper-confirmed that the strongest statistical relationships were found with the smoking behaviour of best friends. ${ }^{89}$

School related variables have drawn less attention. Nutbeam and Aaro ${ }^{10}$ used the school related questions in the HBSC survey to examine negative attitudes towards school and education and their relationship to cigarette smoking. Their data suggested a strong association between regular smoking and alienation from school, consistent for boys and girls in the different cultures and social organisations represented by participating countries. As school is an important source of social learning, we expected school variables to be relevant in predicting smoking behaviour. 
The question arose as to what degree the same predictors of smoking are found in non-western cultures. If we found the same prominent predictors, or similar patterns, in smoking onset and maintenance, we could assume that smoking prevention campaigns developed and used in the West could be useful in other countries as well, at least when they target the same variables. Research suggests that predictors of smoking are subject to crosscultural variations. Landrine et $a l^{11}$ reported that the amount of smoking among blacks, Asians, Hispanics, and whites was different. Moreover, differences for predicting smoking were found. Smoking among peers was the best predictor of smoking for white adolescents (accounting for $23.5 \%$ of the variance) but accounted for only $15 \%$ of the variance for young Hispanics, $9.6 \%$ of the variance for young Asians, and none of the variance for young blacks. A study in Japan among second grade students of a senior high school revealed that smoking behaviour proved to be the best predictor of smoking behaviour 3.5 years later. ${ }^{12}$ Attitudes towards men's smoking, sex, and smoking in subjects' families were also related. These four variables explained $35 \%$ of the variance in smoking behaviour. A study among schoolchildren aged 11 years in Hong Kong showed that believing that their parents will not interfere with their smoking, living with family members who do smoke, and having a positive attitude towards smoking were all factors predictive of smoking. ${ }^{1}$

This paper describes patterns of smoking among adolescents in central Java, Indonesia. In particular, we are interested in determinants of smoking behaviour, and changes in determinants across age groups. Although age was recognised as an important variable in the onset and maintenance of smoking behaviour, few studies have assessed how age modifies the value of predictive models, and how determinants may vary across age. We hypothesised that the influence of peers was not the same for all (adolescent) age groups. This may be an important issue for developing anti-smoking campaigns directed at adolescents.

\section{Method}

SUBJECTS

The data reported stem from the HBSC survey in Indonesia. This survey collected data on a broad range of health behaviours in adolescents, one aim being to direct health promotion initiatives. Details about the adaptation of the HBSC in Indonesia-which is still experimental-are described elsewhere. ${ }^{13}$ Semarang, where this study was conducted, is the commercial capital of central Java. It is a harbour town with a population of 1.5 million people, situated on the north coast of central Java.

The target populations of the HBSC survey were 11,13 , and 15 year olds. For the study in Semarang 17 year olds were also included because smoking behaviour starts later in Indonesia than in most western countries. In the research protocol for the HBSC survey ${ }^{14}$ sampling with school classes as clusters was recommended. To ensure the adequate representation of subpopulations and to overcome the lack of an adequate sampling frame, this procedure had to be modified. Because of differences expected for type of school (private or governmental) and district (urban or rural), these variables were stratified. Unlike most European countries, there were no computerised lists of school classes. The latest updated school directories available for elementary schools dated from 1994 (Kantor Wilayah Departemen Pendidikan dan Kebudayaan), and for high schools the most recent directory was from 1990.

The first step in the sampling procedure was to determine the schools and classes where the targeted age groups could be reached. A pilot study in 1995 revealed that the targeted age groups were in grade six of the elementary school, the second year of junior high school, the first year of senior high school, and in the third year of senior high school. Because children go to school in Semarang at different ages and because of students who have failed, most classes have mixed age groupsconsequently we oversampled. A total of 1026 schools had classes with the targeted age groups.

In the second step the number of classes needed to survey at least 1300 pupils of each age group was determined. In the third step the schools were stratified by age group, public or private status, and by urban or rural district. In the fourth step the number of schools per stratum was determined. The schools were randomly selected within the strata. After the first contact with the schools the classes were randomly selected by the researchers from the list of eligible classes. The total number in the sample was 6276 pupils, with 2410 pupils from 86 elementary schools (86 classes), 1809 pupils from 33 junior high schools (47 classes) and 2057 from 30 senior high schools (53 classes).

Eighteen per cent of the original sampled schools had to be replaced by other schools (randomly selected) for different reasons (schools did not exist any more; some remote schools were impossible to contact; some schools refused to participate).

All students present at the time of data collection were given the questionnaire by research assistants, to whom they were returned anonymously. There was no follow up of absent pupils.

As the percentage of girls who smoke was very low (only $1.1 \%$ of female respondents smoked at least once a week), we studied smoking in male respondents only. The total number of respondents was 1963. Mean ages for the four age categories were 11.5, 13.5, 15.5 , and 17.4 years, respectively.

MEASURES

The HBSC questionnaire measured a variety of health related behaviours. Core questions were the same for all participating countries and include demographic questions about smoking, alcohol use, seatbelt use, physical 
Table 1 Variables entered in the regression model

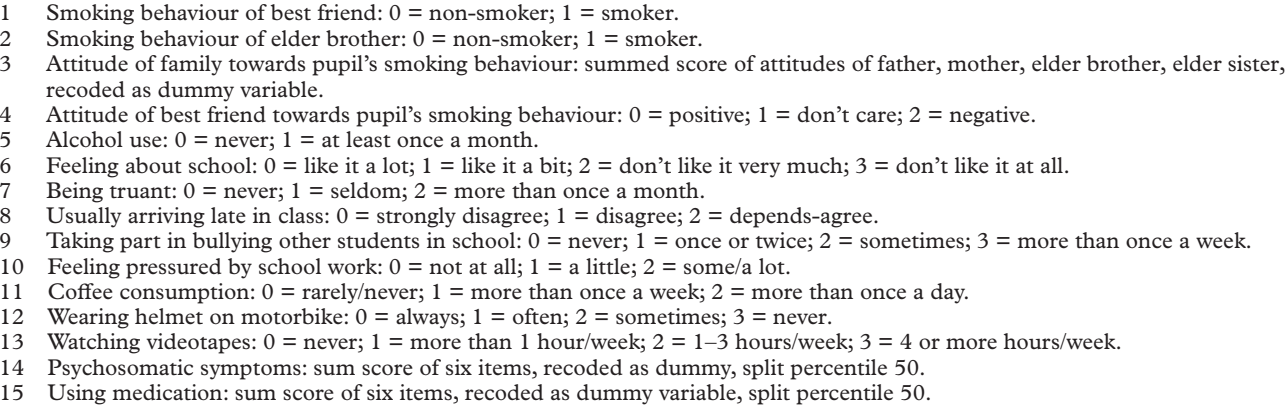

exercise, dietary habits, medication intake, mental and physical health, school life and interpersonal relationships. The adaptation of this questionnaire in Java, Indonesia is still experimental, and corrections are expected in the next study. Shortcomings do not involve smoking and related behaviours that are of concern in this paper, however. ${ }^{13}$

Smoking behaviour was assessed by the question: "How often do you smoke at present?" with four responses: every day; at least once a week, but not every day; less than once a week; and I do not smoke. For this study smoking was defined as at least weekly smoking.

In addition, questions on the age at which the first cigarette was smoked, smoking behaviour, the approval by others of adolescent smoking, and the effect on smoking of the social environment (father, mother, brothers and sisters, best friend, and teachers) were included.

From all available variables in the HBSC core and focus questions, those variables which have been associated with the onset of smoking were selected ${ }^{70}$ for this analysis.

Data were analysed with the statistical software SPSS v.7.5 for Windows 95. We conducted two series of logistic regression analyses to assess the contribution of several variables to the prediction of the binary dependent variable (smoking/not smoking). The regression models were conducted for each age category separately.

The Wald $\chi^{2}$ statistic, the square of the ratio of the regression coefficient to its standard error, was used to evaluate the contribution of a variable entered into a logistic regression model, ${ }^{15}$ together with odds ratios (OR) and $95 \%$ confidence intervals (95\% CI).

First, Spearman correlations were calculated between smoking behaviour and other

Table 2 Prevalence of smoking in male adolescents by age and social environment

\begin{tabular}{lcclr}
\hline & $\begin{array}{l}11 \text { years } \\
n(\%)\end{array}$ & $\begin{array}{l}13 \text { years } \\
n(\%)\end{array}$ & $\begin{array}{l}15 \text { years } \\
n(\%)\end{array}$ & $\begin{array}{l}17 \text { years } \\
n(\%)\end{array}$ \\
\hline $\begin{array}{l}\text { Tried smoking } \\
\text { Regular smoking }\end{array}$ & $126(20.8)$ & $165(29.9)$ & $301(46.3)$ & $76(50.7)$ \\
$\begin{array}{l}\text { Negative attitude of } \\
\text { Father }\end{array}$ & $50(8.2)$ & $86(15.5)$ & $161(24.7)$ & $58(38.7)$ \\
$\begin{array}{l}\text { Mother } \\
\text { Older brother }\end{array}$ & $542(89.6)$ & $467(84.4)$ & $595(91.5)$ & $118(79.2)$ \\
$\begin{array}{l}\text { Older sister } \\
\text { Best friend }\end{array}$ & $566(93.2)$ & $498(90.2)$ & $600(92.4)$ & $128(87.1)$ \\
$\quad$ Teacher & $322(53.5)$ & $287(52.6)$ & $340(53.2)$ & $73(50.0)$ \\
\hline
\end{tabular}

${ }^{\star}$ Regular smoking was defined as at least once a week. behavioural measures for each age group separately. Variables that turned out to be significantly correlated $(p<0.001)$ for at least one age category were included in the logistic regression model. To avoid multicolinearity (and add to the relevance of the model) new variables were computed. For example, the questionnaire includes questions about respondents' use of medications for headaches and stomach aches Since it turned out that these questions are highly intercorrelated, a new variable - general medication intake-was computed by summing all separate medication variables. The same was done for psychosomatic complaints, smoking behaviour of "significant others", and attitude of significant others towards respondent's smoking. The newly computed variables were then recoded into dummy variables, and their correlation with smoking behaviour compared with the original variables. The most relevant (highest correlation) was selected to avoid colinearity of the predictor variables. For example, smoking behaviour of elder brothers and best friends was entered separately in the model, not the 'grouping' variable, smoking behaviour of others. Fifteen variables were entered in the regression model (table 1).

Actual smoking behaviour of the adolescents-defined as smoking daily or weekly - was the dependent variable used in the logistic regression. Next, the variable was recoded into a dummy variable (smoking/nonsmoking).

\section{Results}

PREVALENCE OF SMOKING

A total of $20.8 \%(n=126)$ of the 11 year olds male respondents and $50.7 \%(n=76)$ of the 17 year olds had ever tried smoking. The percentage of smokers (defined as smoking a cigarette at least once a week) dramatically increased between the ages of 11 and 17, from $8.2 \%(n=50)$ to $38.7 \%(n=58)$ (table 2$)$.

Students were asked in which situations they smoke most (table 3 ). Of the current smokers, $60.9 \%(\mathrm{n}=30)$ of 11 year olds and $86.6 \%(\mathrm{n}=$ 50 ) of 17 year olds reported smoking more in the company of friends, compared with $22.0 \%$ $(\mathrm{n}=11)$ and $63.8 \%(\mathrm{n}=37)$ when feeling lonely; $28.0 \%(n=14)$ and $67.2 \%(n=39)$ when having problems; $24.0 \%(\mathrm{n}=12)$ and $24.2 \%(\mathrm{n}=14)$ when with the family, and $14.0 \%(n=7)$ and $69.0 \%(n=40)$ at a party. 
Table 3 Regular smokers * smoking more in specific situations by age

\begin{tabular}{lccrr}
\hline & $\begin{array}{l}11 \text { years } \\
n(\%)\end{array}$ & $\begin{array}{l}13 \text { years } \\
n(\%)\end{array}$ & $\begin{array}{l}15 \text { years } \\
n(\%)\end{array}$ & $\begin{array}{l}17 \text { years } \\
n(\%)\end{array}$ \\
\hline With friends & $30(60.9)$ & $67(77.9)$ & $131(81.2)$ & $50(86.6)$ \\
When lonely & $11(22.0)$ & $30(34.9)$ & $74(45.9)$ & $37(63.8)$ \\
When having problems & $14(28.0)$ & $27(31.4)$ & $83(51.5)$ & $39(67.2)$ \\
With family & $12(24.0)$ & $16(18.6)$ & $16(10.0)$ & $14(24.2)$ \\
At a party & $7(14.0)$ & $29(33.7)$ & $92(57.5)$ & $40(69.0)$ \\
\hline
\end{tabular}

$\star$ Regular smoking was defined as at least once a week.

Table 4 Predictive value of regression model for each age category, with variance explained $\left(R^{2}\right)$, goodness of fit, and correctly classified smokers

\begin{tabular}{llllll}
\hline Age (years) & $R^{2}$ & Goodness of fit & $\begin{array}{l}\text { Correctly } \\
\text { classified smokers } \\
(\%)\end{array}$ & Smokers (\%) & $n$ \\
\hline 11 & 19.8 & 612 & 66.67 & 8.2 & 608 \\
13 & 31.3 & 500 & 73.75 & 15.5 & 554 \\
15 & 40.6 & 662 & 79.49 & 24.7 & 651 \\
17 & 53.0 & 143 & 80.00 & 38.7 & 150 \\
\hline
\end{tabular}

$R^{2}$ was measured by Nagelkerke statistics.

These results confirm the influence of peers on smoking behaviour. With growing age, reported smoking behaviour increased in all situations, except within the family. Surprisingly, $24.0 \%$ of the 11 year old boys reported smoking more when within the family. This was the same as among 17 year olds.

Respondents perceived their social environment as mostly negative towards their smoking. Most respondents reported that their parents would disapprove of their smokingslightly less among the 17 year olds (mother: $87 \%$; father: $79 \%$ ). Older sisters and brothers were perceived as relatively more permissive towards smoking. Teachers' attitudes to smoking were somewhere in between: $80.6 \%$ of the 11 year olds and $75.0 \%$ of the 17 year olds thought that their teachers would disapprove of their smoking. Best friends had the most permissive attitude towards smoking. Surprisingly, age did not affect the respondents' perceptions about the attitudes of significant others towards their smoking behaviour, except for best friends. Regarding parents', teachers', and siblings' negative attitudes, the differences between the 11,13 , and 15 year old boys were negligible. For the oldest group we found a marginal decline in negative attitude towards pupil's smoking. For best friends the percentage of people who disapprove dropped from $55.9 \%$ to $34 \%$.

FACTORS ASSOCIATED WITH SMOKING

As the analysis was based on survey results, the effects found must be interpreted as associations and not as causal relations.

As expected, the effects found in the regression models differed substantially in each age category. Smoking status could be predicted more accurately in the older age groups. The variance (Nagelkerke statistics) increased from $19.8 \%$ for 11 year olds to $53 \%$ for the 17 year olds (table 4). The goodness of fit decreased from 612 to 143 . When taking a cut point that takes into account the percentage of smokers within a certain age category, the percentage of correctly predicted smokers remained quite stable over the age categories, ranging from $66 \%$ to $80 \%$.
FACTORS ASSOCIATED WITH SMOKING BY AGE Among 11 year old boys, only their best friends' smoking had a significant effect on their own smoking behaviour (Wald $\chi^{2}=$ $14.61, \mathrm{p}<0.001)$. Young people whose best friend smoked were almost four times more likely to smoke themselves than those whose best friend did not smoke (table 5). None of the other 14 variables entered in the regression model significantly contributed to the prediction of smoking behaviour.

For 13 year old boys, six variables had a significant effect on smoking behaviour: best friend's smoking behaviour (Wald $\chi^{2}=14.59$, $\mathrm{p}=0.0001$ ), helmet wearing on a motorbike (Wald $\chi^{2}=8.43, p=0.004$ ), truanting (Wald $\chi^{2}=7.41, \mathrm{p}=0.006$ ), elder brother's smoking behaviour (Wald $\chi^{2}=5.60, \mathrm{p}=0.018$ ), coffee consumption (Wald $\chi^{2}=5.30, p=0.021$ ), and best friend's attitude towards respondent's smoking (Wald $\chi^{2}=4.53, \mathrm{p}=0.033$ ). The OR of being a smoker increased to 2 for youngsters who had an older brother who smoked. The OR increased to 3 if their best friend smoked.

Among 15 year olds, best friends' smoking behaviour became even more important (Wald $\left.\chi^{2}=61.06, p<0.000\right)$. The OR of being a smoker increased by a factor of 7.33 when their best friend smoked. Other significant effects were truanting (Wald $\chi^{2}=12.97, \mathrm{p}=0.0003$ ), and coffee consumption (Wald $\chi^{2}=7.95, \mathrm{p}=$ 0.0048). The family's attitude towards smoking (Wald $\chi^{2}=2.98, p=0.084$ ) and best friends' attitude towards smoking (Wald $\chi^{2}=$ 3.04, $\mathrm{p}=0.081$ ), alcohol use (Wald $\chi^{2}=3.30$, $\mathrm{p}=0.0694)$, and psychosomatic complaints (Wald $\chi^{2}=2.79, \mathrm{p}=0.0949$ ) were moderately associated with smoking, but these relations were not statistically significant.

For 17 year olds, the effect of peers slightly decreased: best friends' smoking behaviour (Wald $\chi^{2}=7.19, \mathrm{p}=0.007$ ) and best friends' attitudes towards the respondents' smoking (Wald $\chi^{2}=6.43, p=0.0112$ ) were still significant predictors of smoking behaviour. Having a smoking friend increased the OR of being a smoker by a factor of 4.5. This decreased effect of peer smoking among 17 year olds is difficult to interpret because of the large $95 \%$ CI (1.5 to 13.4). Arriving late in class was associated with smoking behaviour (Wald $\chi^{2}=4.11, \mathrm{p}=$ 0.043). Alcohol use was also moderately related to smoking, but not significantly (Wald $\left.\chi^{2}=3.41, \mathrm{p}=0.065\right)$.

After the initial analysis, variables with significance levels of $p \leqslant 0.01$ for at least one age group were entered into a second series of logistic regression models. Alcohol was included in the model, as for the three oldest age groups $(13,15$, and 17 year olds), alcohol use came very close to significance $(\mathrm{p}=0.053$, 0.069 , and 0.065 respectively). We hypothesised that with the elimination of less important variables, the effect of alcohol use in the regression model might become more prominent. Although only eight variables remained in the models, the variance found for all age categories was only slightly less compared with the first regression analysis. The relative importance of most of the 
Table 5 Odds ratios (OR) and confidence intervals (CI) for smoking/non-smoking respondents according to selected predictors by age group

\begin{tabular}{|c|c|c|c|c|c|c|c|c|c|c|c|c|}
\hline & \multicolumn{3}{|c|}{11 years $(n=608)$} & \multicolumn{3}{|c|}{13 years $(n=554)$} & \multicolumn{3}{|c|}{15 years $(n=651)$} & \multicolumn{3}{|c|}{17 years $(n=150)$} \\
\hline & OR & $95 \% C I$ & $p$ & $O R$ & $95 \% C I$ & $p$ & $O R$ & $95 \% C I$ & $p$ & $O R$ & $95 \% C I$ & $p$ \\
\hline Liking school & 0.74 & $0.28-1.94$ & 0.543 & 0.55 & $0.29-1.05$ & 0.072 & 1.44 & $0.93-2.23$ & 0.102 & 1.25 & $0.39-4.00$ & 0.706 \\
\hline Brother smokes & 1.84 & $0.91-3.71$ & 0.089 & 2.01 & $1.13-3.60$ & 0.018 & 1.50 & $0.94-2.37$ & 0.086 & 1.98 & $0.76-5.16$ & 0.164 \\
\hline Best friend smokes & 3.81 & $1.92-7.57$ & 0.000 & 2.95 & $1.69-5.14$ & 0.000 & 7.33 & $4.45-12.09$ & 0.000 & 4.48 & $1.50-13.41$ & 0.007 \\
\hline Attitude of best friend & 0.74 & $0.49-5.62$ & 0.134 & 0.68 & $0.47-0.97$ & 0.033 & 0.76 & $0.56-1.03$ & 0.081 & 0.42 & $0.22-0.82$ & 0.011 \\
\hline Attitude of family & 1.22 & $0.79-2.19$ & 0.694 & 1.21 & $0.66-2.22$ & 0.532 & 1.53 & $0.94-2.48$ & 0.084 & 1.94 & $0.74-5.09$ & 0.180 \\
\hline Alcohol use & 1.66 & $0.51-1.27$ & 0.413 & 2.57 & $0.99-6.71$ & 0.053 & 1.68 & $0.96-2.93$ & 0.069 & 3.09 & $0.93-10.26$ & 0.065 \\
\hline Playing truant & 1.31 & $0.79-2.20$ & 0.294 & 1.72 & $1.16-2.54$ & 0.006 & 1.83 & $1.32-2.54$ & 0.000 & 1.13 & $0.54-2.33$ & 0.749 \\
\hline Late at school & 0.80 & $0.51-1.27$ & 0.340 & 1.44 & $0.94-2.20$ & 0.094 & 1.28 & $0.93-1.76$ & 0.124 & 2.25 & $1.03-4.92$ & 0.043 \\
\hline Bullying & 1.47 & $0.96-2.21$ & 0.073 & 1.22 & $0.88-1.70$ & 0.227 & 1.15 & $0.90-1.46$ & 0.273 & 0.99 & $0.62-1.56$ & 0.949 \\
\hline Pressure of school tasks & 1.52 & $0.92-2.62$ & 0.105 & 1.00 & $0.62-1.62$ & 0.983 & 1.03 & $0.71-1.49$ & 0.888 & 1.47 & $0.63-3.45$ & 0.375 \\
\hline Coffee drinking & 1.39 & $0.94-2.07$ & 0.097 & 1.49 & $1.06-2.10$ & 0.021 & 1.55 & $1.14-2.10$ & 0.005 & 0.83 & $0.43-1.60$ & 0.582 \\
\hline Helmet wearing & 1.16 & $0.84-1.61$ & 0.375 & 1.51 & $1.14-2.00$ & 0.004 & 1.16 & $0.94-1.43$ & 0.155 & 0.68 & $0.43-1.06$ & 0.086 \\
\hline Watching videotapes & 0.85 & $0.56-1.27$ & 0.429 & 1.28 & $0.95-1.73$ & 0.104 & 0.87 & $0.69-1.11$ & 0.266 & 1.56 & $0.97-2.52$ & 0.067 \\
\hline Somatic complaints & 0.84 & $0.43-1.61$ & 0.593 & 1.62 & $0.92-2.85$ & 0.098 & 1.47 & $0.93-2.32$ & 0.095 & 1.88 & $0.73-4.85$ & 0.191 \\
\hline Medication use & 1.81 & $0.90-3.64$ & 0.095 & 1.49 & $0.84-2.64$ & 0.173 & 0.90 & $0.56-1.44$ & 0.665 & 1.03 & $0.40-2.62$ & 0.954 \\
\hline
\end{tabular}

"Smoking" is defined as smoking at least once a week.

variables remained the same. As expected, the effect of alcohol use on smoking behaviour increased, although only for 13 and 17 year olds (Wald $\chi^{2}=4.65, \mathrm{p}=0.03$; Wald $\chi^{2}=5.9$, $\mathrm{p}=0.01)$.

\section{Discussion}

Smoking in Indonesia is a serious problem and justifies more efforts to stop it, or better, to prevent youngsters from starting to smoke. The best targets are probably 11-15 year olds. By the age of 17, adolescents' smoking behaviour has not only dramatically increased but also will probably be incorporated in their lifestyles, which makes it more difficult to quit smoking.

The prevalences found in this study are difficult to compare with the results of other studies in the same region because of different age groups or lack of information on age groups or research design. ${ }^{416}$ In our study no questions were asked on the type of cigarettes smoked. The adult population in Indonesia traditionally smokes clove cigarettes (kretek; $60 \%$ tobacco and $40 \%$ ground cloves). However in a qualitative study on the smoking behaviour of young people in Semarang we found that young people preferred ordinary, filter cigarettes.

Apparently, peers were the most dominant mediators in the onset (and maintenance) of smoking behaviour, and this was true for all age categories. Although in this study the interpretation of the effect of peers was limited to the associations found, and cannot be interpreted as a causal effect, these results do not differ from studies in other southeast Asian or Western countries. Peer influence seemed almost universal. For 11 year olds, best friends' smoking behaviour was the only significant effect.

Having an older brother who smoked also had a significant effect on smoking behaviour in 13 year olds, but became less important for older adolescents. Parental smoking behaviour and attitudes towards respondents' smoking did not have a significant effect.

In general we found that school related items played a less important role in the onset of smoking behaviour than expected. Bullying, pressure of school tasks, and liking school were not significantly related with smoking. Arriving late in class was related to smoking behaviour but only among 17 year olds. Truanting was a significant predictor of smoking only among 15 year olds. The lesser effect of school related items may be genuine in Semarang but the results should also be treated with caution. The survey was school administered and the tendency to answer school related items in a socially desirable way may be a notable factor. The main rule in Javanese society is to avoid loss of face for all concerned, and to avoid open conflict. It is very important to sense what will please the other party and to answer accordingly. Although this compliance effect may also play a role in other variables, the questions about the school were, in a school context, very sensible.

Other variables also turned out to be less important: watching videotapes, psychosomatic complaints, and medication use were not associated with smoking behaviour. The fact that ownership of videotape recorders is very limited in Java and that the opportunities to watch videotapes outside the home is limited could have had an effect on the results.

The effects of intrapersonal measures and the relationship with physical complaints have been assessed in only a few studies in Western countries and the results found cannot be generalised. ${ }^{17}$ In this study an indirect measure of physical complaints was used (medication use), and it may be that physical complaints in the ages studied do not lead to medication use.

Contrary to what we expected, ${ }^{18}$ alcohol use was not strongly associated with smoking behaviour, although for 13 and 17 year olds, significant probability values were found. The fact that only a few people use alcohol probably affects the statistical value of the data. Although cigarette smoking is socially acceptable, this is not the case for alcohol use. Indonesia is predominantly a Muslim country and drinking alcohol is strictly forbidden in the Koran. Alcohol use is considered to be a much more (social and religious) deviant behaviour than smoking.

Not surprisingly, as the number of smokers among younger adolescents is still relatively low, prediction of smoking among this group (11 year olds) seemed to be less reliable than among older adolescents. This is particularly regrettable, for knowledge about smoking predictors for this group would allow us to 
influence young adolescents before they take up smoking. This suggests more research is needed, with younger adolescents (11 year olds) as the primary target group.

Insofar as we intended to present a picture of smoking behaviour among school aged children, one shortcoming of this study was that data were only collected in schools. An estimated $15 \%$ of all school aged children were not reached. This group might be especially at risk for smoking and other risk behaviours. Further research should therefore target this population. The study was also limited by its cross-sectional nature-a longitudinal study, following young students until they are 17 years old (or more) could give a definitive answer on the determinants of smoking behaviour of young people in Indonesia. The dataset used was also limited to the variables included in the protocol of the HBSC survey. A recent longitudinal study provided evidence that tobacco promotional activities are causally related to the onset of smoking in young people. ${ }^{19}$ Further studies in Semarang on the smoking behaviour of young people should also include information on the exposure to tobacco advertising and promotional activities and on the receptivity to these activities.

This study was part of a joint cooperation between the Catholic University of Semarang, Indonesia and the State University of Ghent, Belgium. The project was supported by the Flemish InterUniversity Council (VLIR) and funded by the Belgium Department for Developmental Cooperation (ABOS). The Department for Developmental Cooperation (ABOS). The promotor was Paulette Van Oost, head of the department of behavioural therapy and psychological consultation. The study EURO cross-national study.

1 Peters J, Hedley JJ, Lam TH, et al. Factors influencing smoking behavior in Hong Kong primary school children targets for prevention. Asia-Pacific $\mathcal{F}$ Public Health 1995; 8:102-8.

2 Collishaw NE, Lopez AD. Prevalence of cigarette smoking in developing countries (editorial). Tobacco Control 1995; $4: 327$.
3 Ganiwijaya T, Sjukrudin E, De Backer G, et al. Prevalence of cigarette smoking in a rural area of West Java, Indonesia. Tobacco Control 1995; 4:335-7.

4 Kristanti CM. Generasi muda dan masalah perilaku berisiko. Majalah Kesehatan Masyarakat Indonesia 1995; 23:92-7. (In Indonesian)

5 Melby JN, Conger RD, Conger KJ, et al. Effects of parental behavior on tobacco use by young male adolescents. 7 Marriage Fam 1993;55:439-54.

6 Bertrand LD, Abernathy TJ. Predicting cigarette smoking among adolescents using cross-sectional and longitudinal among adolescents using cross-sectional and

7 Reid DJ, McNeil AD, Glynn TJ. Reducing the prevalence of smoking in youth in Western countries: an international overview. Tobacco Control 1995;4:266-77.

8 Aaro LE, Wold B, Kannas L, et al. Health behaviour in schoolchildren: a WHO cross-national survey-a presentation of philosophy, methods and selected results of the first survey. Health Prom 1986;1:17-33.

9 Wold B, Aaro LE, eds. Health behaviour in school-aged children. A WHO cross-national survey. [Summary of publications form the first survey (1983-84) and the second survey (1985-86).] Bergen, Norway: University of Bergen Research Center for Health Promotion; Copenhagen, Denmark: World Health Organisation, Regional Office for Europe, 1990.

10 Nutbeam D, Aaro LE. Smoking and pupil attitudes towards school: the implications for health education with young people. Results from the WHO study of health behaviour among schoolchildren. Health Educ Res 1991;4:415-21.

11 Landrine H, Richardson JL, Klonoff EA, et al. Cultural diversity in the predictors of adolescent cigarette smoking: the relative influence of peers. F Behav Med 1994;17:331

12 Kawabata T, Orlandi MA, Takahashi H, et al. Prediction of smoking behavior in Japanese young adults. Health Educ Res 1992;7:437-42.

13 Smet B, Maes L, De Clercq L, et al. The health behaviour in school-aged children study in Indonesia: methodological problems in cross-cultural research. Health Prom Int 1999; 14:7-16.

14 Wold B, Aaroe LE, Smith C. Health behaviour in school-aged children. A WHO cross-national survey. Research protocol for the 1993-94 survey. Bergen, Norway: University of Bergen Research Centre for Health Promotion, 1993.

15 Sarason IG, Mankowski ES, Peterson AV, et al. Adolescents' reasons for smoking. $\mathcal{F}$ Sch Health 1992;62:185-90.

16 Melby JN, Conger RD, Conger KJ, et al. Effects of parental smoking on tobacco use by young male students. $\mathcal{f}$ Marriage Fam 1993;55:439-54.

17 Conrad KM, Flay BR, Hill D. Why children start smoking cigarettes: predictors of onset. Br f Addict 1992;87:1711-

18 Nutbeam D, Aaroe L, Wold B. The lifestyle concept and health education with young people. Results from a WHO international survey. World Health Stat $O$ 1991;44:55-61.

19 Pierce JP, Choi WS, Gilpin EA, et al. Tobacco industry promotion of cigarettes and adolescent smoking. $\mathscr{f} A M A$

\section{A hundred years ago}

"The drunkard does not compel you to drink, the opium-eater to eat opium, but the smoker makes you smoke, nay more, visibly inhale the very vapor just ejected from his own mouth."

Matthew Woods. Some of the minor immoralities of the tobacco habit. "FAMA" 1899;32:683-7. 